\title{
The Responses of Injection Pressure and Fracture Width during Height Extension in Sand-Mud Interbed Reservoirs in the Dongsheng Gas Field
}

\author{
Di Wang $\mathbb{D}^{1,2,3}$ Haibo Wang, ${ }^{1,2,3}$ Fengxia Li, ${ }^{1,2,3}$ Fuhu Chen, ${ }^{4}$ and Xinchun Zhu ${ }^{4}$ \\ ${ }^{1}$ State Key Laboratory of Shale Oil and Gas Enrichment Mechanisms and Effective Development, Beijing 100083, China \\ ${ }^{2}$ National Energy Shale Oil Research and Development Center, Beijing 100083, China \\ ${ }^{3}$ SINOPEC Petroleum Exploration and Production Research Institute, Beijing 102206, China \\ ${ }^{4}$ Petro-Engineering Research Institute of Sinopec North China Oil and Gas Branch, Zhengzhou 450006, China
}

Correspondence should be addressed to Di Wang; wangdi304@126.com

Received 9 September 2021; Accepted 12 October 2021; Published 28 November 2021

Academic Editor: Yu Wang

Copyright (c) 2021 Di Wang et al. This is an open access article distributed under the Creative Commons Attribution License, which permits unrestricted use, distribution, and reproduction in any medium, provided the original work is properly cited.

The Dongsheng gas field is characterized by low porosity and low permeability. Its principal producing reservoir is the $\mathrm{H}-1$ zone, belonging to the Lower Shihezi Formation. Sand is the main lithology in the H-1 zone, while mud interlayers are also well developed in a vertical direction. As a result, the reservoir is a sand-mud interbed, which brings difficulty to fracture height extension. In order to understand the process of fracture height growth in a sand-mud interbed reservoir and obtain the responses of injection pressure and fracture width during a hydraulic fracturing, a hydromechanical-coupled model is established. Mud interlayers are fully considered and a cohesive zone model is adopted to deal with fracture propagation. Numerical results show that the fracture extends quickly to the sand-mud interface after initiation and breaks through rather than propagating along the interfaces. Pressure and width both increase continuously when fracture propagates in the mud interlayer. High-viscosity and high-injection rates are helpful for the fracture to break through the mud interlayer, especially at an early period. When the mud interlayers are asymmetric, pressure and width fluctuate several times once fracture propagates inside and breaks through the mud interlayer. Perforations close to the thinner mud interlayer can increase the fracture width and reduce fracturing risks.

\section{Introduction}

The Dongsheng gas field belongs to the Erdos basin, which locates in North China. The principal producing reservoir of the Dongsheng gas field is Lower Shihezi Formation, especially the $\mathrm{H}-1$ zone. Without too many complex structures, the formation is flat overall and has a good continuity horizontally. The thickness of the $\mathrm{H}-1$ zone ranges from $10 \mathrm{~m}$ to $50 \mathrm{~m}$ and there are multiple gas formations in the vertical direction.

The main sedimentary system of the $\mathrm{H}-1$ zone includes the alluvial fan and braided stream. As a result, the lithology is very complicated, including pebbly sandstone, coarse sandstone, medium sandstone, fine sandstone, siltstone, and dark mud. Particularly, the coarse sandstone, medium sandstone, and fine sandstone are the main reservoirs.
Due to the influence of river channel migration and the variation of the flood scale, barely permeable and impermeable interlayers are well developed vertically in the $\mathrm{H}-1$ zone [1]. The most common interlayers in the $\mathrm{H}-1$ zone are mud and property interlayers [2], as shown in Figure 1. The lithologies of mud interlayers have the characteristic of high clay content, such as pure mudstone, silty mudstone, and argillaceous siltstone, while the lithologies of the property interlayer are primarily siltstone or argillaceous fine siltstone. Compared with the mud interlayer, the property interlayer has higher porosity and permeability but they are still below the lower limit of the effective reservoirs. In this paper, only mud interlayers are considered to investigate its influence on fracture height growth. The thickness of the mud interlayer varies widely from several centimeters to 


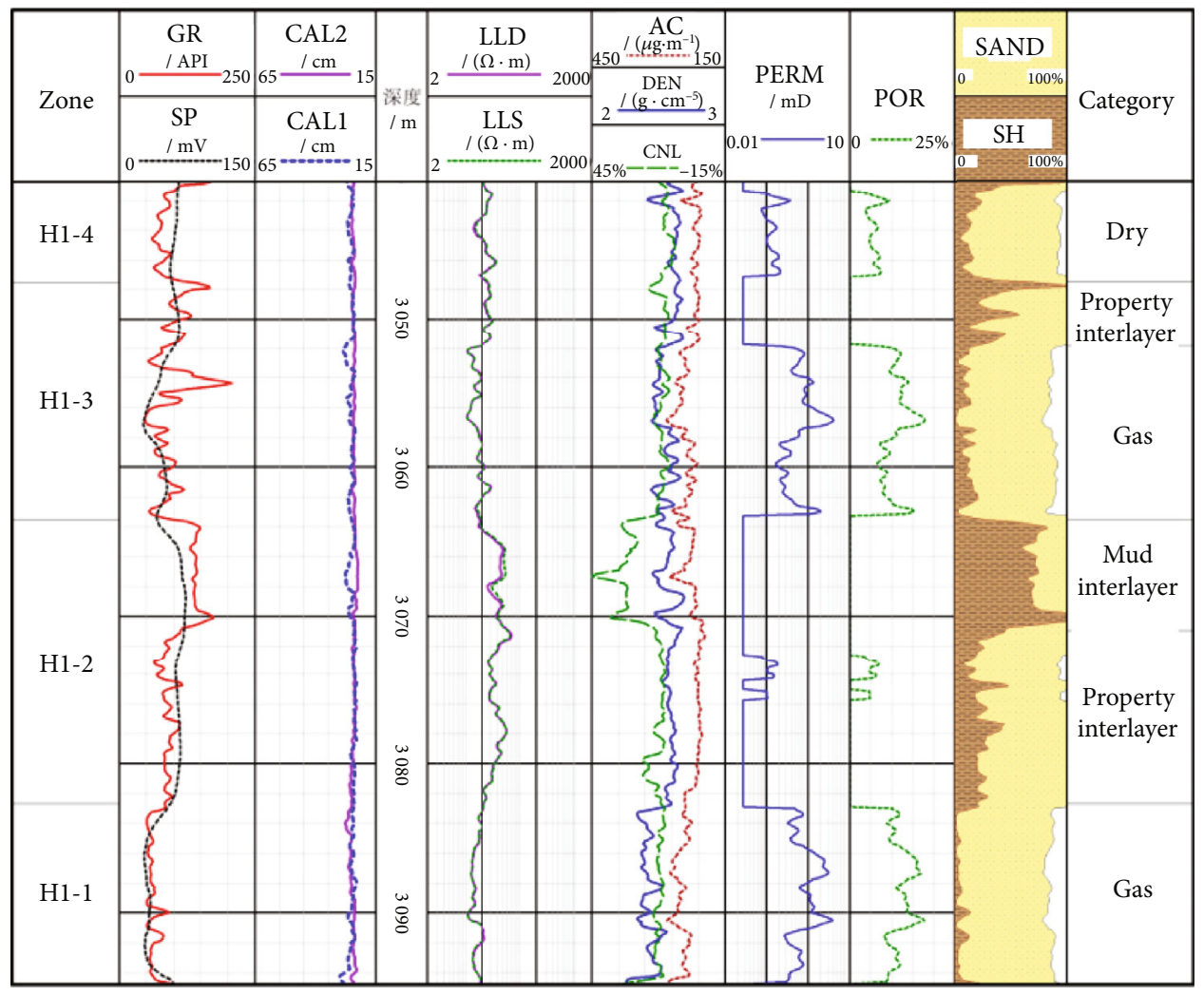

Figure 1: The characteristics of the H-1 zone in the Dongsheng gas field [3].

$25 \mathrm{~m}$. The thick mud interlayers can be treated as caps which can prevent fracture propagation.

The lithologies of gas reservoirs are also complex, including coarse sandstone, medium sandstone, and fine sandstone. The porosity ranges from $5.0 \%$ to $16.9 \%$ and the permeability is mainly in the range from $0.3 \mathrm{mD}$ to $0.9 \mathrm{mD}[4,5]$. Both porosity and permeability are very low, and hydraulic fracturing is the most effective way to develop the Dongsheng gas field. Therefore, it is especially important to understand fracture height growth and specify the responses of injection pressure and fracture width during fracture propagation. In this paper, a hydromechanical coupled numerical model about fracture height extension is established based on the reservoir characteristics in the Dongsheng gas field taking into account of mud interlayers and stress difference. The numerical results illustrate the process of fracture height growth and are instructive to the design of a hydraulic fracturing.

\section{Model}

Classic linear elastic fracture mechanics (LEFM) is a widely used method to evaluate fracture propagation. When the stress intensity factor (SIF) at fracture tip is larger than fracture toughness which is an inherent property of the solid material, fracture initiates and propagates $[6,7]$. At the fracture tip, there is a stress singularity which is described by SIF in LEFM. But the SIF is usually difficult to obtain and this stress singularity bothers numerical simulations at times.
Instead of using the classic LEFM to deal with an elastic crack tip region where stress singularity exists, the cohesive zone model [8-11] assumes the existence of a fracture process zone (where the rock has yielded or experienced microcracking) in front of the material crack tip, which is governed by a traction-separation law. The stress singularity at the crack tip is avoided in cohesive zone models through this constitutive law. In this way, the cohesive zone model provides an alternative approach to explicitly simulate the fracture process near the tip and is often applied in modeling hydraulic fracturing.

2.1. Cohesive Zone Model. In this work, we use a hydromechanical model utilizing the finite element method with a special zero-thickness interface element based on the cohesive zone model which has shown its effectiveness in previous work [12-15].

Indeed, there are numerous kinds of T-S models $[16,17]$ and the classic bilinear traction-separation criteria [18-20] are used in this paper, as shown in Figure 2. These criteria evaluate the stress ratios between a given stress value and the peak nominal stress value in each of the three directions and is based on a quadratic combination of all three ratios [21], as shown in equation (1) as follows:

$$
\left(\frac{\left\langle t_{n}\right\rangle}{t_{n}^{0}}\right)^{2}+\left(\frac{t_{s 1}}{t_{s 1}^{0}}\right)^{2}+\left(\frac{t_{s 2}}{t_{s 2}^{0}}\right)^{2}=1,
$$




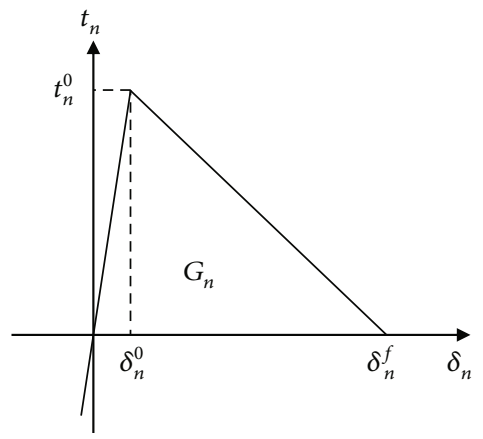

(a)

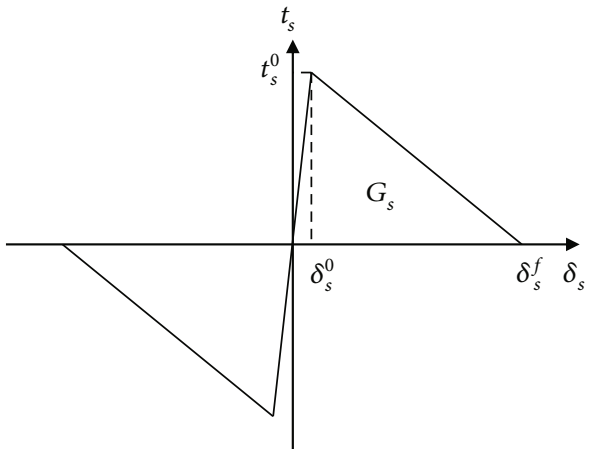

(b)

FIgURE 2: Bilinear traction-separation constitutive law for (a) tensile and (b) shear failure of the cohesive element.

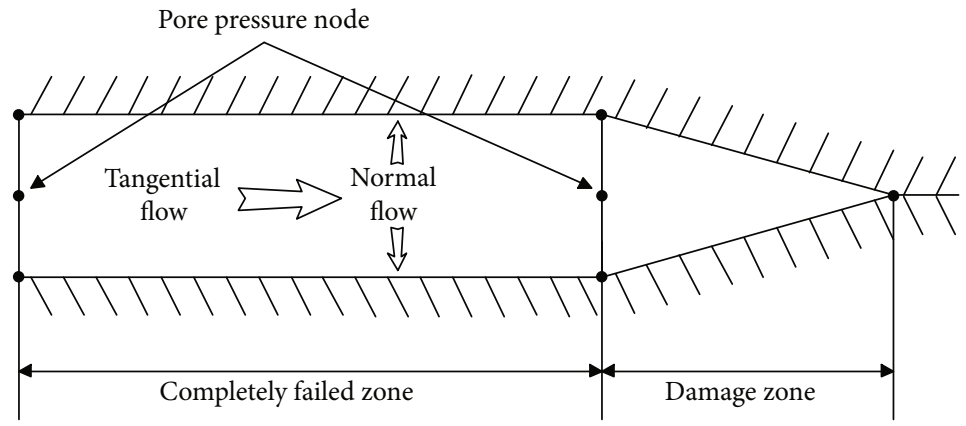

FIGURE 3: Sketch of fluid flow within cohesive elements.

where $t_{n}, t_{s 1}, t_{s 2}$ are nominal stress, first direction shear stress, and second direction shear stress. $t_{n}^{0}, t_{s 1}^{0}, t_{s 2}^{0}$ represent the peak values of the nominal stress when the deformation is either purely normal to the interface or purely in the first or the second shear direction. \langle\rangle is the Macaulay bracket with the usual interpretation $\left\langle t_{n}\right\rangle=t_{n}$ if $t_{n} \geq 0$ and $\left\langle t_{n}\right\rangle=0$ if $t_{n}<0$.

The fracture initiation and propagation are natural outcomes of a cohesive zone model. After cohesive element debonds, fluid flows in the hydromechanical interface and transmit pressure on the cohesive element, as shown in Figure 3.

2.2. Fluid Flow. We assume that the rock surrounding the hydraulic fractures is permeable. Leakoff and poroelastic effects are considered during fracture propagation. Therefore, the fluid flow can be divided into three processes: flow inside the fracture, leakage through fracture, and seepage in the matrix.

As to incompressible fluid, flow in the hydromechanical cohesive interface is derived from the fluid mass balance equation:

$$
\frac{\partial w}{\partial t}+\nabla \cdot \mathbf{q}+q_{L}=Q(t) \delta(x, y)
$$

where $w$ is normal opening, which equals to the displacement jump in the normal direction of the interface, $\mathbf{q}$ is the longitudinal fluid flux, $q_{L}$ is fluid leakoff, $Q(t)$ is the source term, and $\delta(x, y)$ is the Dirac delta function.

The lubrication equation for fluid flow between parallel plates is used to simulate flow in the fracture. It is given as follows:

$$
\mathbf{q}=\mathbf{v} w=-\frac{w^{3}}{12 \mu} \nabla p
$$

where $\mathbf{v}$ is the average fluid velocity over the cross-section of the hydraulic fracture, $\mu$ is the viscosity of the Newtonian fluid, and $p$ is fluid pressure inside the fracture.

Combining equations (2) and (3), we obtain the equation for fluid flow in a hydraulic fracture:

$$
\frac{\partial w}{\partial t}-\nabla \cdot \frac{\mathrm{w}^{3}}{12 \mu} \nabla p+q_{L}=Q(t) \delta(x, y)
$$

For the seepage in the matrix, according to the theory of poroelasticity, the continuity equation utilized in numerical formulation is as follows [22]:

$$
\nabla\left(\frac{k}{\gamma_{l}}\left(\nabla p_{0}-\mathbf{b}_{l}\right)\right)+\mathbf{m}^{T} \dot{\varepsilon}=0
$$

where $k$ is matrix permeability coefficient, $\gamma_{l}$ is specific weight of liquid, $p_{0}$ is the pore pressure, $\mathbf{b}_{\mathbf{l}}$ is the body force 


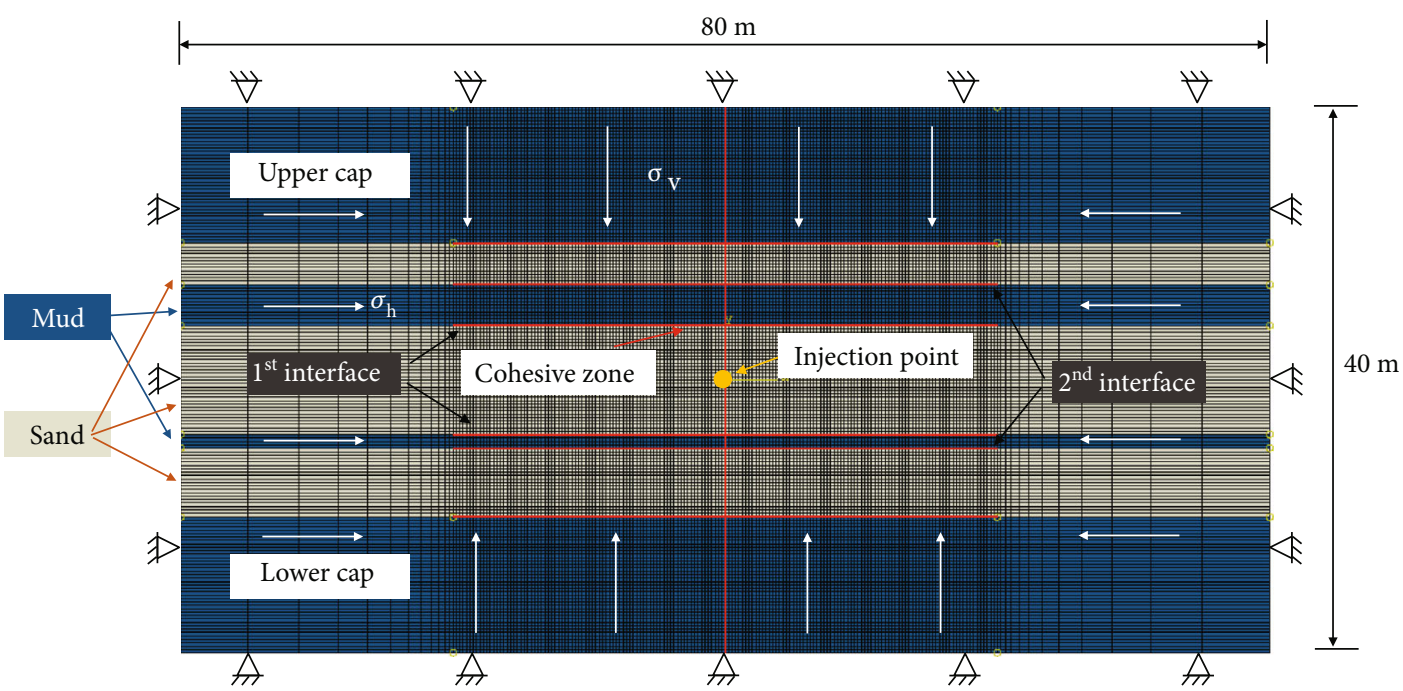

Figure 4: Sketch of the sand-mud interbed numerical model.

of the liquid phase, $\varepsilon$ is the strain tensor, and $\mathbf{m}=[1,1$, $1,0,0,0]$.

In addition, fluid leakage through the fracture wall can be expressed by the following:

$$
q_{L}=C_{L}\left(p-p_{0}\right)
$$

where $C_{L}$ is leakoff coefficient.

2.3. Rock Deformation. The governing equations of rock deformation include the equilibrium equation, geometric equation, and constitutive equation $[23,24]$. The equilibrium equation is written as follows:

$$
\nabla \cdot \sigma+\mathbf{b}=0
$$

where $\boldsymbol{\sigma}$ is stress tensor and $\mathbf{b}$ is body force vector of the rock.

According to Boit's theory, effective stress can be expressed as follows:

$$
\boldsymbol{\sigma}^{\prime}=\boldsymbol{\sigma}+\alpha p_{0} \mathbf{I}
$$

where $\sigma^{\prime}$ is the effective stress tensor, $\alpha$ is the Biot coefficient, and $\mathbf{I}$ is the Kronecker delta.

The geometric equation is written as follows:

$$
\boldsymbol{\varepsilon}=\frac{1}{2}\left(\nabla \mathbf{u}+\nabla \mathbf{u}^{T}\right)
$$

The constitutive relationship can be expressed as

$$
\begin{aligned}
& \boldsymbol{\sigma}=\lambda \varepsilon_{\mathrm{vol}} \boldsymbol{\delta}+2 G \boldsymbol{\varepsilon}-C \zeta \boldsymbol{\delta}, \\
& p=C \varepsilon_{\mathrm{vol}}-M \zeta,
\end{aligned}
$$

where $\lambda$ and $G$ are the Lamé parameters of the porous material, while $C$ and $M$ are additional elastic moduli required to describe a two-phase medium. $\varepsilon_{\mathrm{vol}}$ is the volumetric strain. $\zeta$ is a strain parameter describing the volumetric deformation
TABLE 1: Rock mechanical properties for simulation.

\begin{tabular}{lccc}
\hline Property & $\begin{array}{c}\text { Fine } \\
\text { sand }\end{array}$ & $\begin{array}{c}\text { Coarse } \\
\text { sand }\end{array}$ & $\begin{array}{c}\text { Mud cap/ } \\
\text { interlayer }\end{array}$ \\
\hline Young's modulus $(\mathrm{GPa})$ & 35 & 59 & 20 \\
Poisson's ratio & 0.2 & 0.25 & 0.3 \\
Tensile strength $(\mathrm{MPa})$ & 2.5 & 2.4 & 7.51 \\
Cohesion $(\mathrm{MPa})$ & 36 & 6.4 & 13 \\
Friction angle $\left(^{\circ}\right)$ & 19 & 48 & 18 \\
\hline
\end{tabular}

TABLE 2: Basic parameters for simulation.

\begin{tabular}{lcc}
\hline Category & Sand & Mud cap/interlayer \\
\hline Damage displacement $(\mathrm{m})$ & $10^{-4}$ & $10^{-4}$ \\
Porosity (\%) & 10 & 1 \\
Permeability $(\mathrm{mD})$ & 1 & 0.1 \\
Leakoff coefficient $(\mathrm{m} / \mathrm{Pa} \cdot \mathrm{s})$ & $10^{-13}$ & $10^{-14}$ \\
Vertical stress $(\mathrm{MPa})$ & 75 & 75 \\
Minimum horizontal stress $(\mathrm{MPa})$ & 60 & 64 \\
Pore pressure $(\mathrm{MPa})$ & \multicolumn{2}{c}{30} \\
Fluid viscosity $(\mathrm{mPa} \cdot \mathrm{s})$ & \multicolumn{2}{c}{$6 \times 10^{-4}$} \\
Injection rate $\left(\mathrm{m}^{2} / \mathrm{s}\right)$ & \multicolumn{2}{c}{}
\end{tabular}

of the fluid relative to that of the solid. $\delta$ is the Kronecker function.

2.4. Model Construction. In this study, a sand-mud interbed model is established. In Figure 4, the whole model is $80 \mathrm{~m}$ $\times 40 \mathrm{~m}$ in size. The thicknesses of upper and lower caps are both $10 \mathrm{~m}$. Between the upper and lower caps, there are two asymmetric mud interlayers as the thicknesses of the upper and lower interlayers are $3 \mathrm{~m}$ and $1 \mathrm{~m}$, respectively. The first and second interfaces are defined according to the sequence when hydraulic fracture is encountered. Injection point is right at the center of the model. Cohesive zones are presented along the fracture height path and sand-mud 

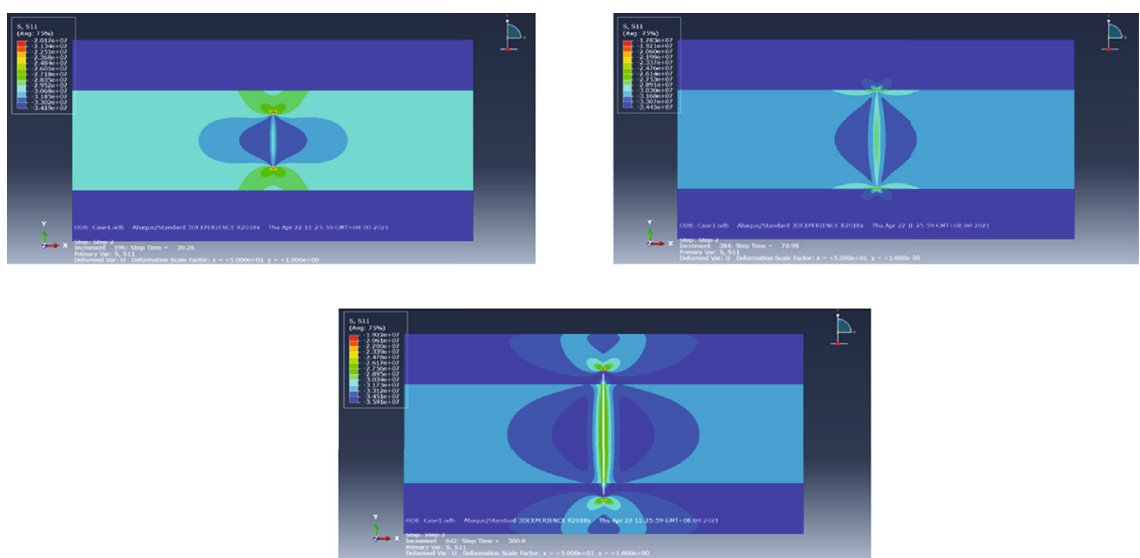

FIGURE 5: Height growth process in the reservoir without mud interlayers.

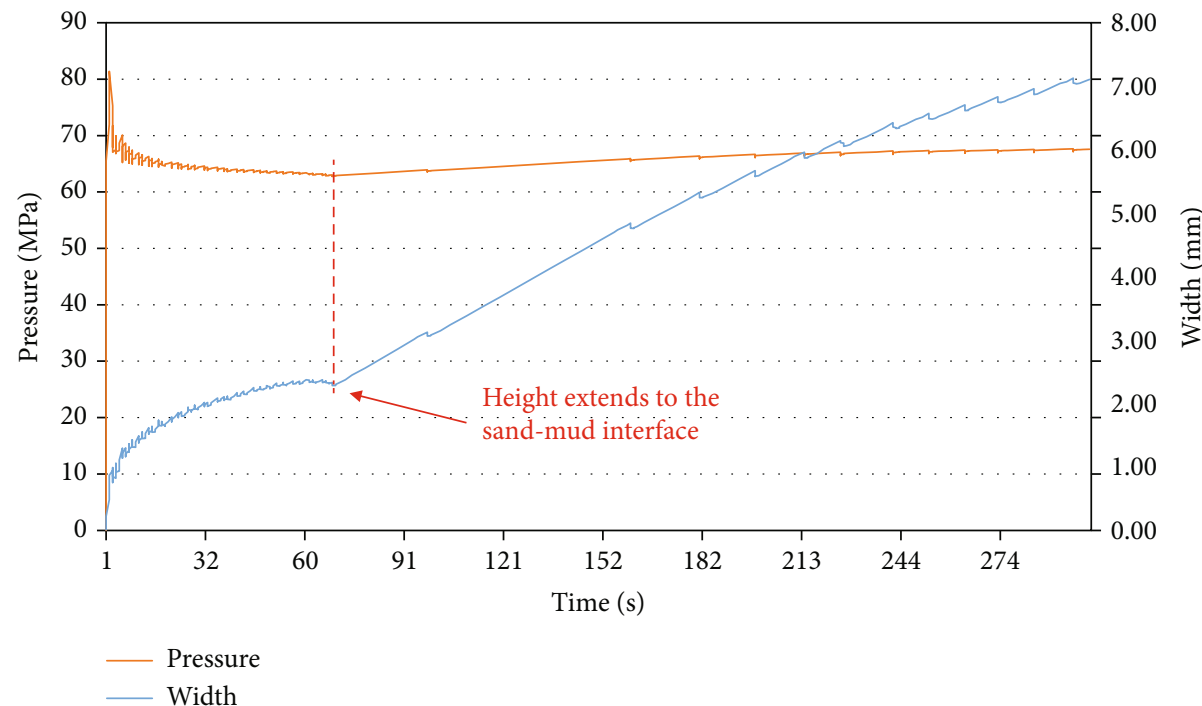

FIgURE 6: The responses of injection pressure and fracture width during height growth in the reservoir without mud interlayers.

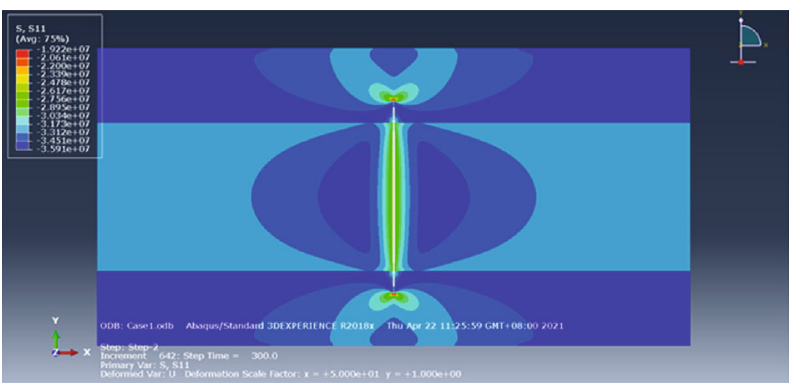

(a) Fine sand

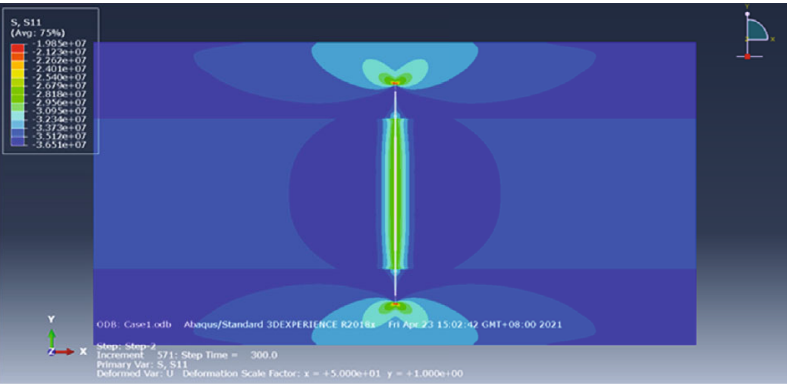

(b) Coarse sand

FIGURE 7: The influence of the rock property on height growth.

interfaces in order to investigate fracture height growth behavior at sand-mud interfaces.

Rock mechanical parameters adopted in numerical simulations are shown in Table 1, and they are all obtained from experimental measurements using downhole cores from the Dongsheng gas field. In order to investigate the influence of rock mechanics on fracture height growth, both fine sand and coarse sand are tested.

Table 2 includes basic parameters except for rock mechanics. The reservoir parameters are all used according to the Dongsheng gas field so that the numerical models are consistent with the actual reservoir conditions as much as possible. 

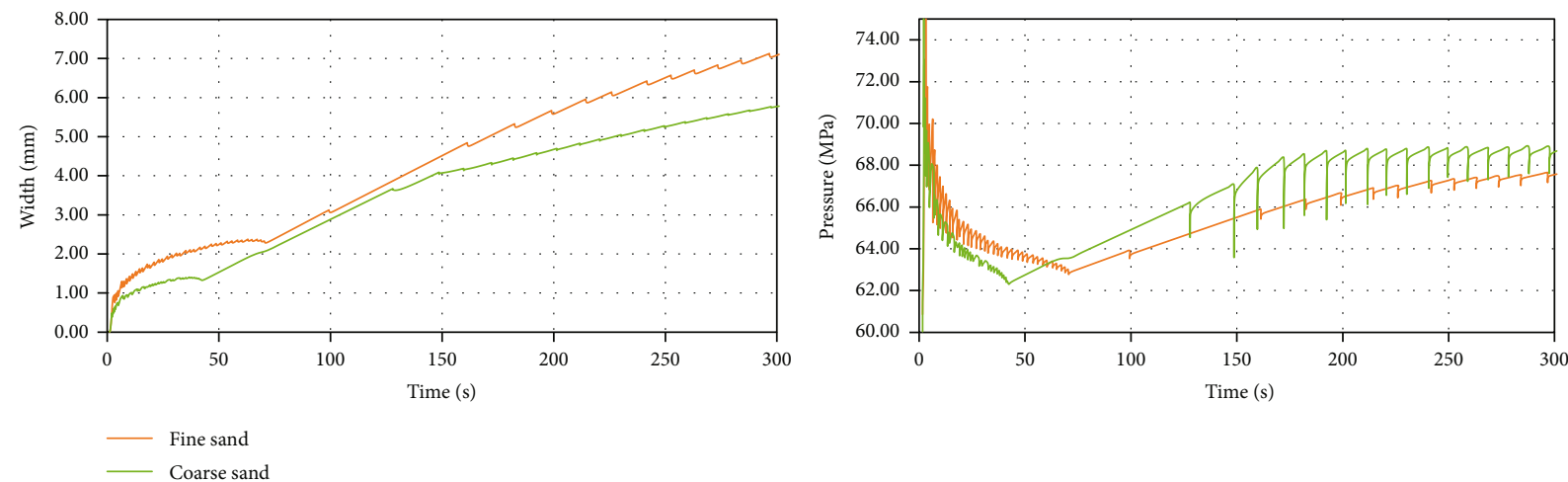

FIGURE 8: The influence of the rock property on injection pressure and fracture width.

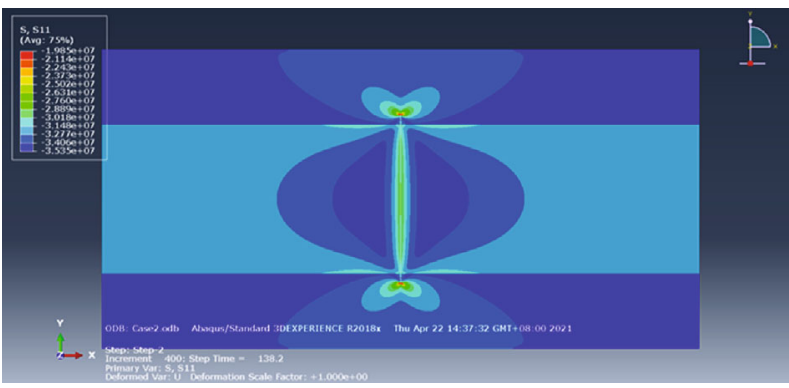

(a) $4 \mathrm{MPa}$

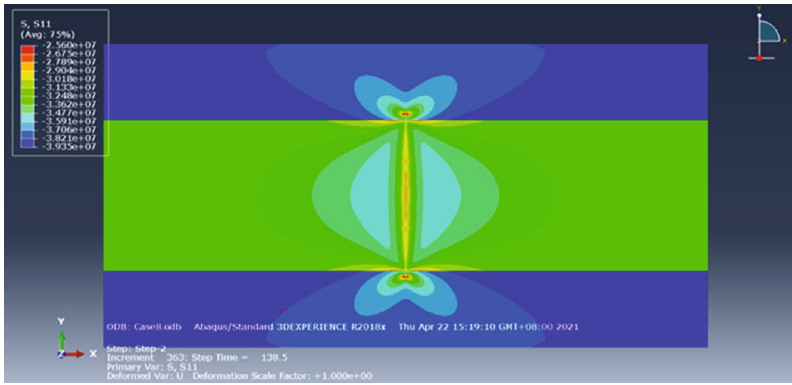

(b) $8 \mathrm{MPa}$

Figure 9: The influence of minimum horizontal stress difference on height growth.
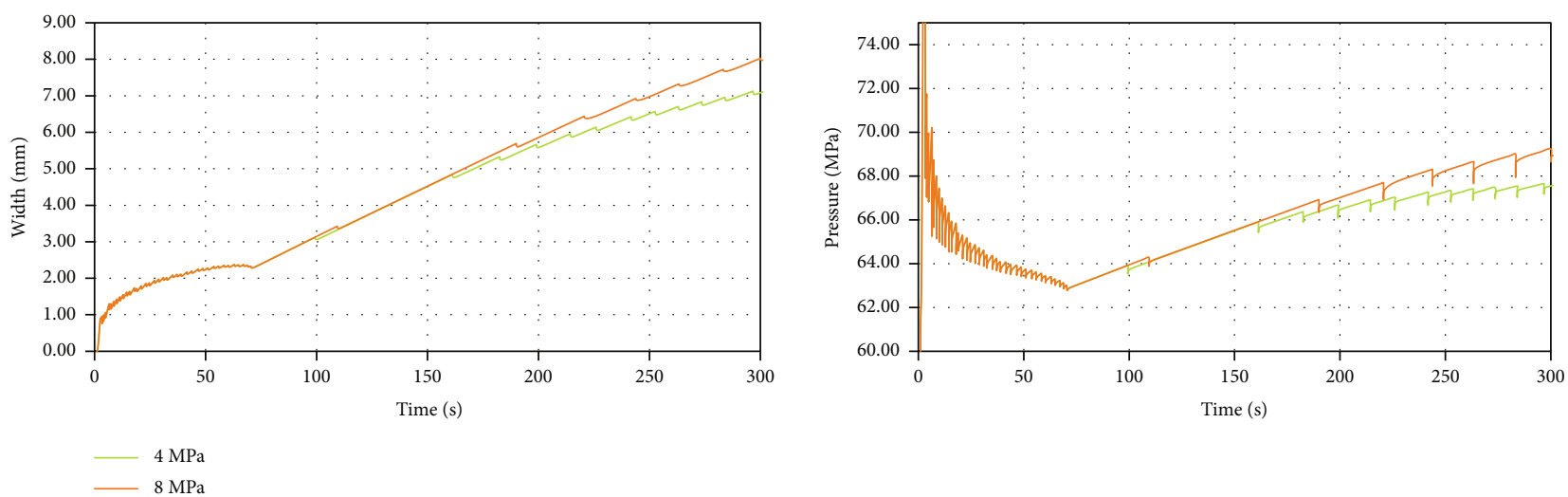

FIGURE 10: The influence of minimum horizontal stress difference on injection pressure and fracture width.

\section{Results}

In order to clearly illustrate about fracture height growth in sand, we first study the cases neglecting mud interlayers. Afterwards, we consider two symmetric mud interlayers whose thicknesses are both $3 \mathrm{~m}$. In the end, two asymmetric mud interlayers as shown in Figure 4 are taken into account which is in accordance with the characteristics of the Dongsheng gas field.

3.1. Reservoirs without Mud Interlayers. Figure 5 shows fracture height extension in sand. At the sand-mud interface, the fracture passes through directly and extends to the cap. This is because the reservoir shear strength is much larger than tensile strength and tensile failure dominates. Besides, when fluid wants to flow into the interface, it needs to overcome overburden stress or vertical stress, which is very difficult because the reservoir is deeper than $3000 \mathrm{~m}$. But at the interface, it still can be observed that stress discontinuity exists.

Figure 6 clearly shows the fracture width and pressure responses during propagation. When fracture propagates in sand, pressure drops rapidly after initiation and keeps being smooth, while the width increases slowly. When the fracture reaches the interface and extends in the cap, propagation slows down. As a result, pressure increases and width 

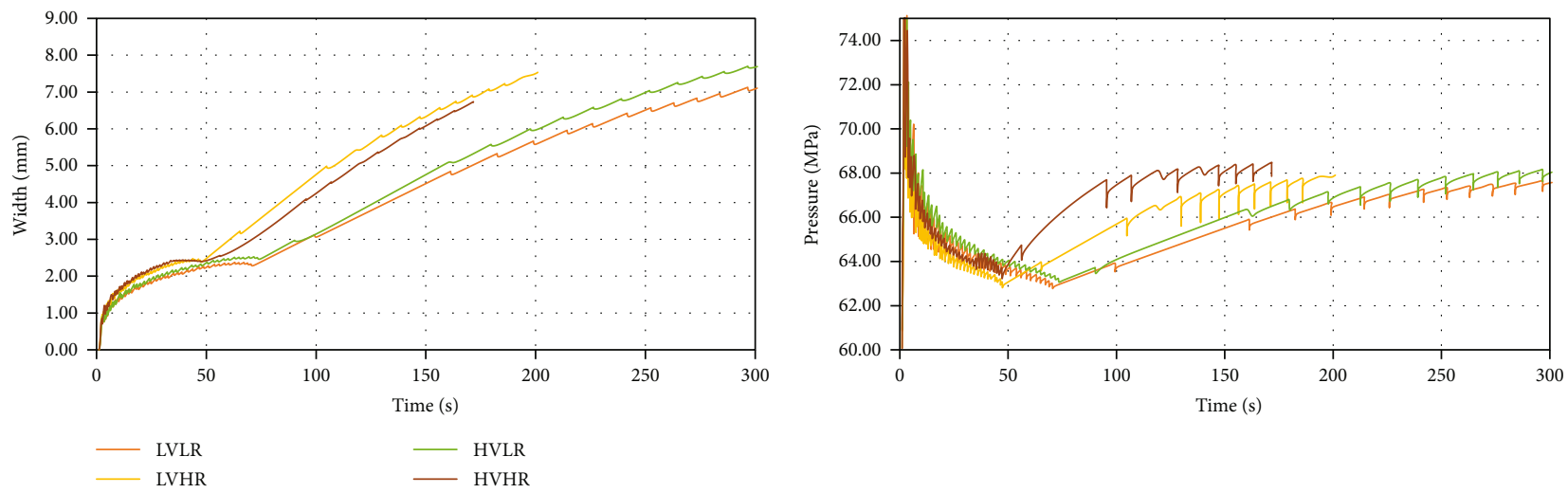

FIGURE 11: The influence of fluid viscosity and injection rates on injection pressure and fracture width.

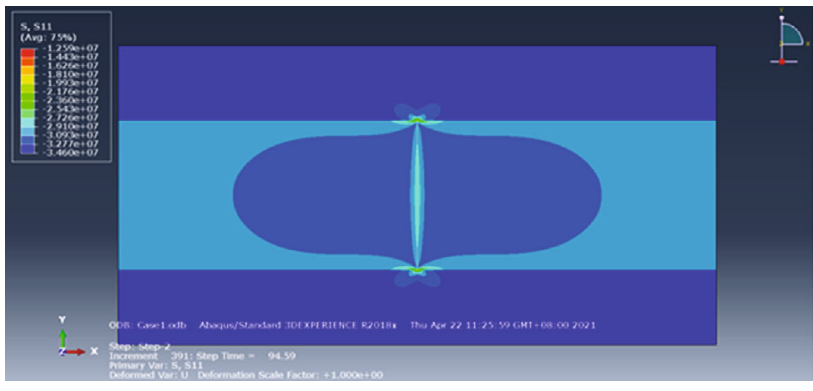

(a) LVLR

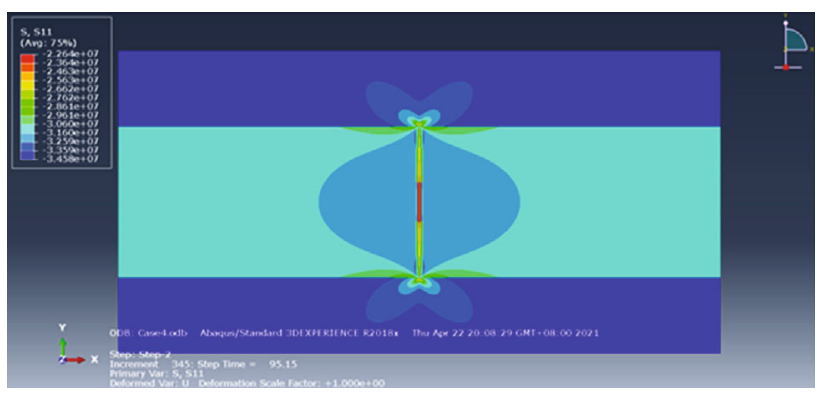

(c) HVLR

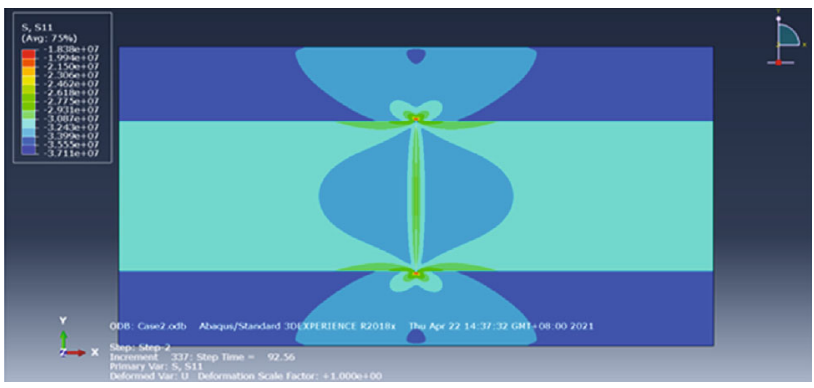

(b) LVHR

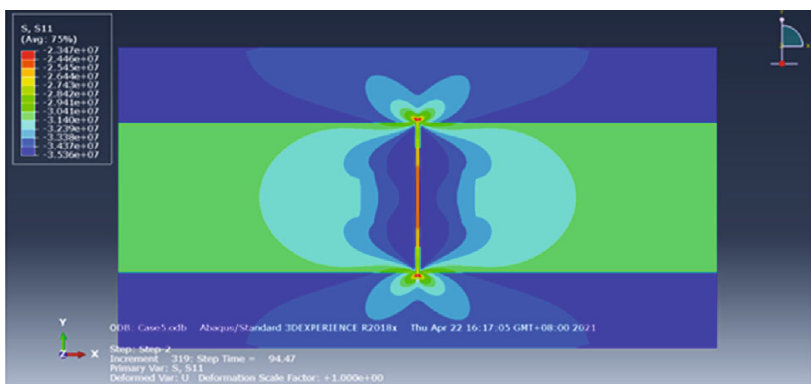

(d) HVHR

Figure 12: The influence of fluid viscosity and injection rates on height growth.

expands. In reality, when pressure is large enough, fracture will propagate horizontally in sand rather than keep the extension in the cap. The zigzags shown in Figure 6 are due to the mesh size used in the numerical model. With smaller meshes, the lines will be much more smooth but the calculation will be tremendous. Therefore, the element size along fracture is $0.25 \mathrm{~m}$ in this model and calculation accuracy is not affected.

3.1.1. The Effect of Sand Property. Figures 7 and 8 show that the fracture propagates in fine and coarse sands, respectively. With a lower tensile strength, the fracture propagates much easier in coarse sand. As a result, pressure is lower and width is smaller. However, when the fracture extends to the interface, pressure in coarse sand increases rapidly and surpasses that in fine sand. This is because the elastic modulus of coarse sand is lower. Under the same condition, fracture width is smaller and the friction along fracture is higher. If the fracture continuously propagates in the cap, it requires higher pressure to overcome friction.

\subsubsection{The Effect of Minimum Horizontal Stress Difference} between Cap and Sand. Before the fracture reaches the interface, the responses of pressure and width are exactly the same. But after fracture extends into cap with higher stress, pressure increases higher and fracture opens wider, as shown in Figures 9 and 10.

3.1.3. The Effects of Fluid Viscosity and Injection Rate. We consider four cases to investigate the influence of fluid viscosity and injection rate on height growth, and they are the low viscosity and low injection rate (LVLR), low viscosity and high injection rate (LVHR), high viscosity and low injection rate (HVLR), and high viscosity and high injection rate (HVHR). Compared with viscosity, it seems that the injection rate has the most influence on fracture width. 


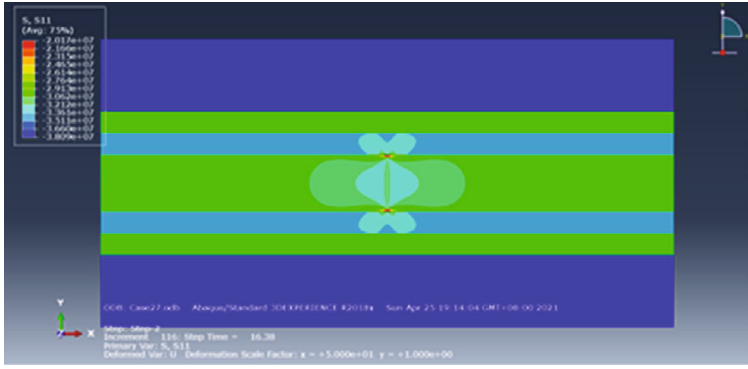

(a) Height extends to the first interface

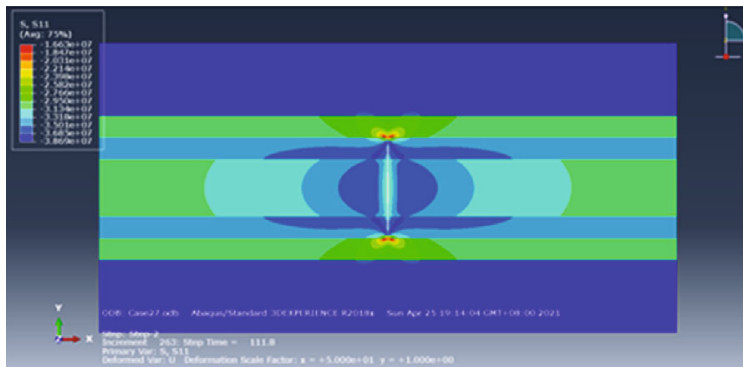

(c) Height extends to the second interface

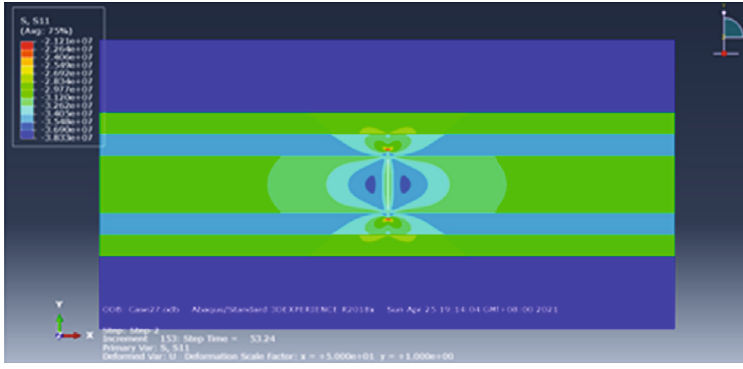

(b) Height breaks through the first interface

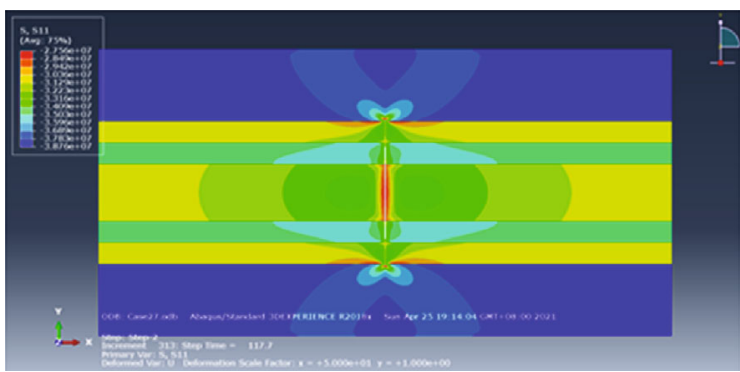

(d) Height breaks through the second interface

FIgURE 13: Height growth process in the reservoir with symmetric mud interlayers.

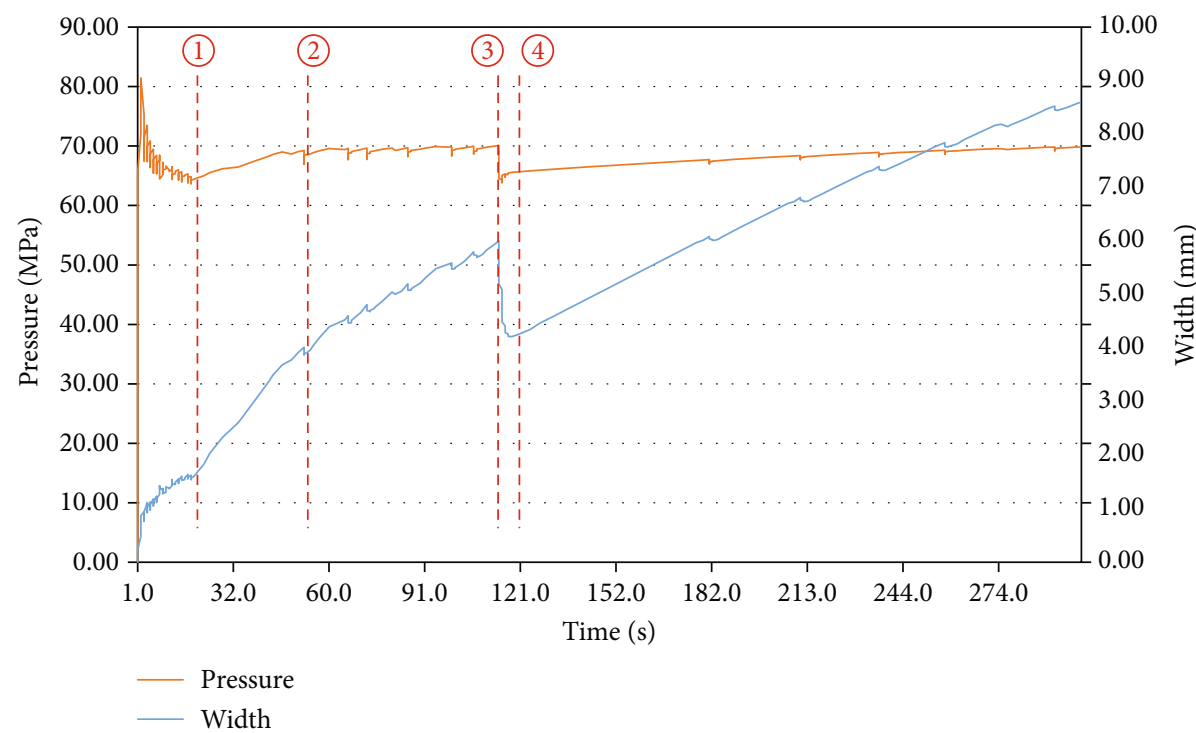

FIGURE 14: The responses of injection pressure and fracture width during height growth in the reservoir with symmetric mud interlayers.

Figure 11 clearly shows that with the high injection rate, fracture width is larger. At the same time, when the fracture extends to the interface, pressure increases rapidly. With higher viscosity, fluid friction along the fracture is higher, as shown in Figures 12(c) and 12(d).

3.2. Reservoirs within Symmetric Mud Interlayers. In this section, two symmetric mud interlayers are inserted in sand. Figure 13(a) shows that fracture extends to the first interface which is corresponding to (1) in Figure 14 when fracture pressure and width are about to increase rapidly. The pressure keeps increasing until (2) when the fracture tip extends into the mud interlayer. While the fracture propagates in the mud interlayer, it seems that pressure no longer increases. Due to the high in situ stress of the mud interlayer, fracture propagation velocity slows down at the moment and friction along the fracture reduces. Therefore, pressure inside the fracture is much more uniform and pressure at the injection point tends to be similar to the pressure at the tip of fracture. As a result, fracture width keeps growing until (3), when the fracture breaks through the second interface and exits the mud interlayer. Afterwards, fracture extends to the interface of sand and cap. But it is a very short period from (3) to (4). This is because when the fracture propagates inside the mud, it requires a higher pressure. When the fracture leaves the mud and goes into the sand, the high 

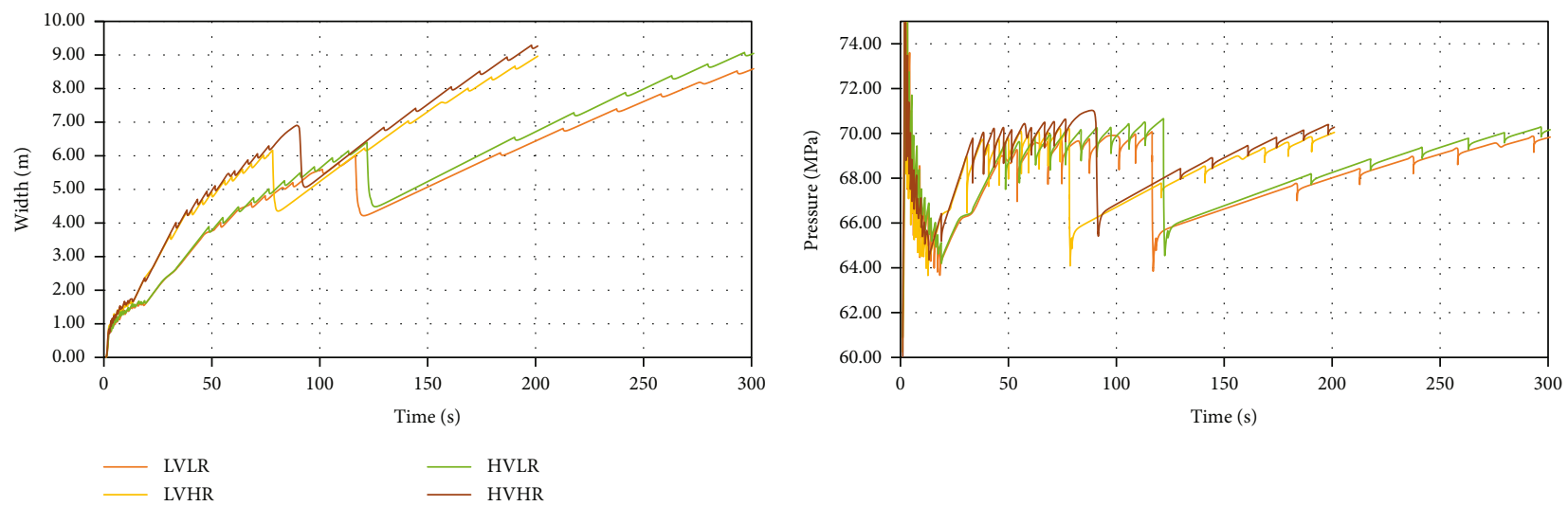

FIGURE 15: The influence of fluid viscosity and injection rates on injection pressure and fracture width.
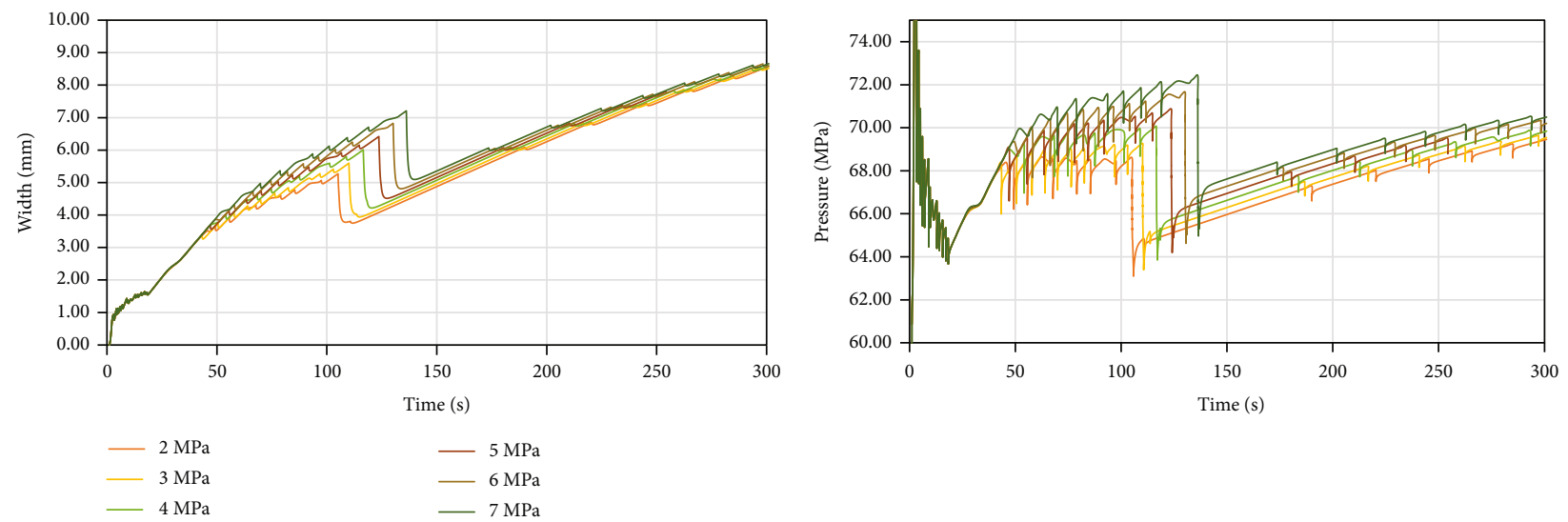

FIGURE 16: The influence of stress difference between the mud interlayer and sand on injection pressure and fracture width.

pressure will accelerate fracture growth. Therefore, the fracture reaches the sand and cap interface quickly as shown in Figure 13(c). In the end, the fracture extends inside the cap and the pressure increases integrally although the pressure at the injection point does not rise up obviously, which results in fracture width increasing continuously.

3.2.1. Effect of Fluid Viscosity and Injection Rate. When the mud interlayers are imbedded in sand, the treatment parameters affect height growth differently. With a high injection rate, the fracture propagates to the first interface and breaks through the mud interlayer quickly. As shown in Figure 15, it seems that under the low fluid viscosity condition, the pressure required to break through mud interlayer is a little bit smaller. This may be resulted from the lower friction when fluid viscosity is low. Under the high viscosity and high injection rate conditions, pressure inside the fracture is the highest. Therefore, when we hope that the fracture breaks through mud interlayers in sand, high viscosity and high injection rate are the best choices especially at the early period.

3.2.2. Effect of Stress Difference between Sand and Mud Interlayers. As shown in Figure 16, before the fracture reaches the first interface, the responses of pressure and

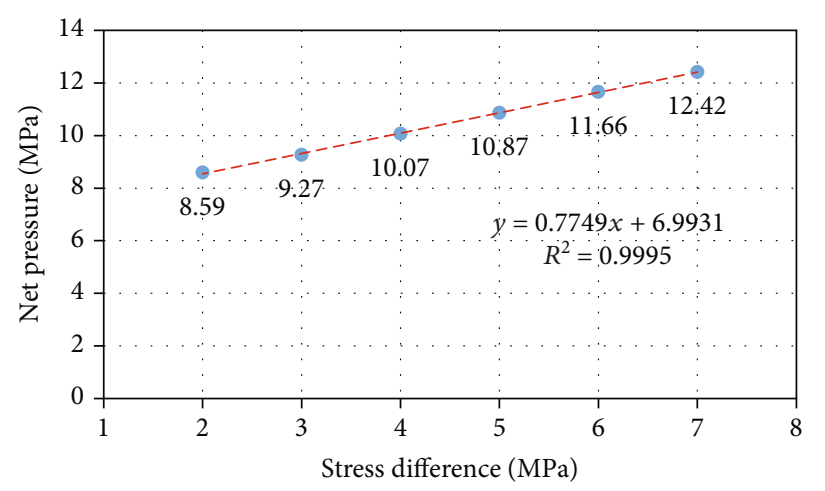

FIGURE 17: The relationship between required net pressure and stress difference.

width are exactly the same. But when the stress difference between sand and mud interlayers increases, the pressure required to break through the mud interlayer is higher and the fracture opens wider. Besides, it takes more time to break through the mud interlayers.

As shown in Figure 17, when the stress difference increases from $2 \mathrm{MPa}$ to $7 \mathrm{MPa}$, the net pressure required to break through the mud interlayer rises up linearly from 8.59 MPa to $12.42 \mathrm{MPa}$. The increment of net pressure, 


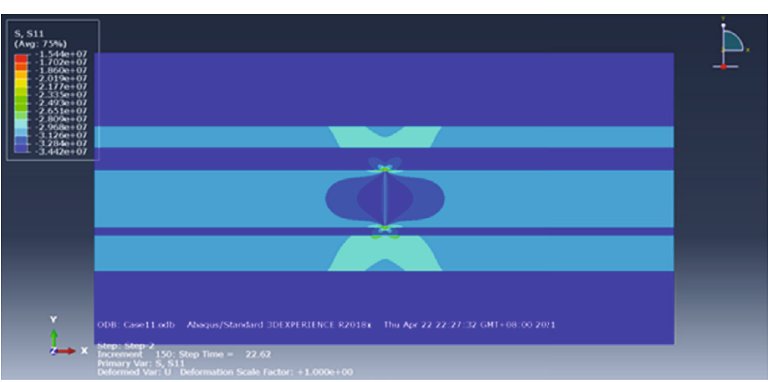

(a)

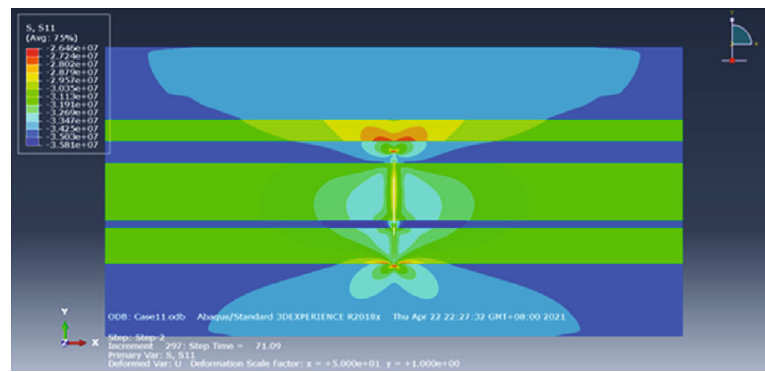

(c)

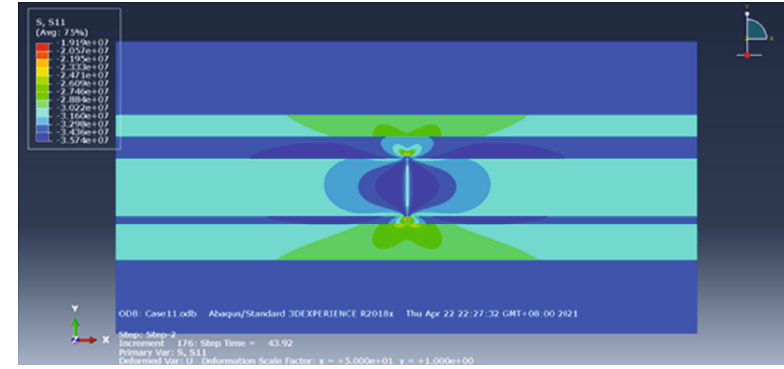

(b)

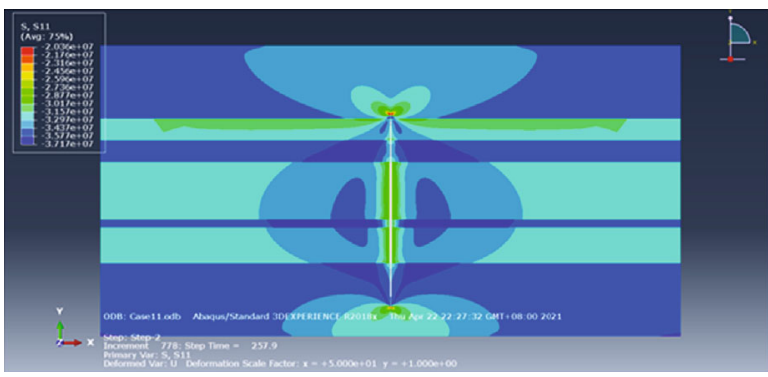

(d)

FIGURE 18: Height growth process in the reservoir with asymmetric mud interlayers: (a) height extends to the first interface, (b) height breaks through the first interface, (c) the lower branch breaks through the mud interlayer and extends to the lower cap, and (d) the upper branch extends to the upper cap.

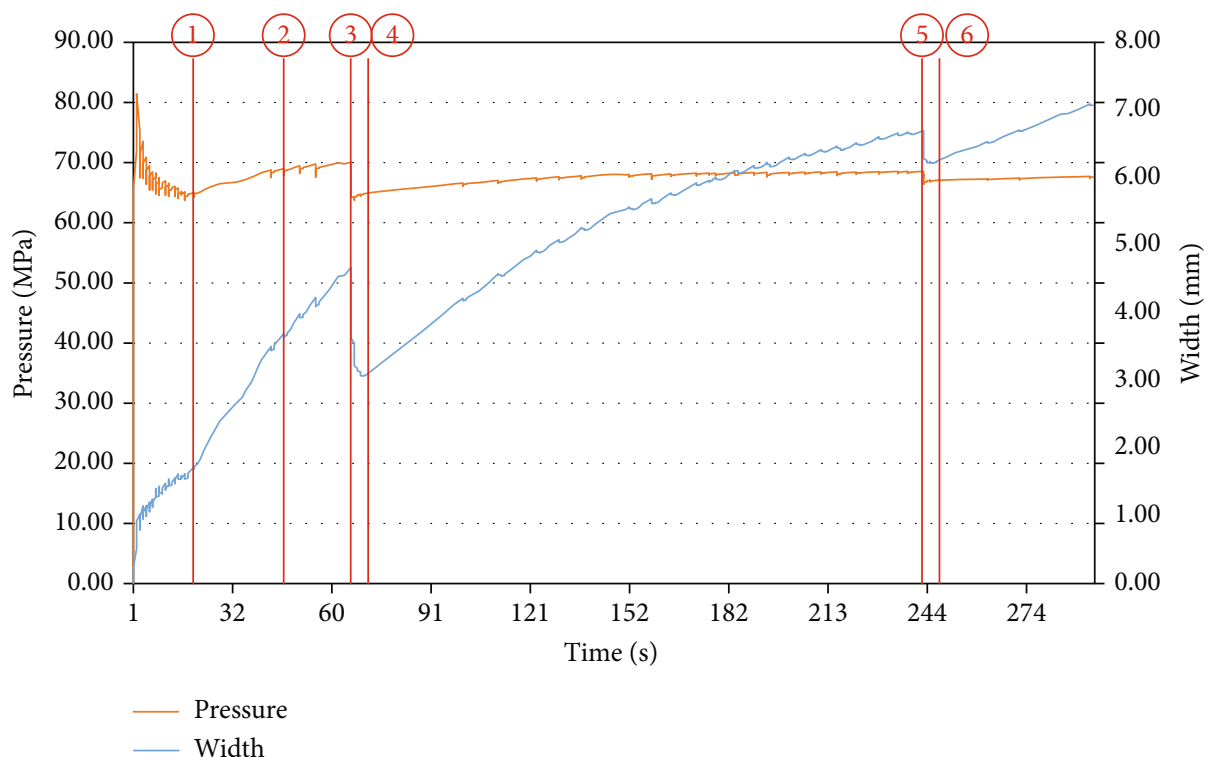

FIGURE 19: The responses of injection pressure and fracture width during height growth in the reservoir with asymmetric mud interlayers.

3.83 $\mathrm{MPa}$, is smaller than the increment of stress difference, $5 \mathrm{MPa}$. This is because when the minimum horizontal stress is large, it becomes much more difficult for fracture to break through. Fracture propagation slows down and fluid friction along the fracture drops. As a result, pressure inside the fracture trends to be uniform and only a small increment of net pressure can keep the fracture propagating.

3.3. Reservoirs within Asymmetric Mud Interlayers. Asymmetric mud interlayers are more common in reality and are considered in this section. The numerical model is the same as shown in Figure 4. The distances between the injection point to the upper and lower first interface are the same.

It can be observed that fracture extension becomes much more complex from that in Figure 18 and the process is divided into several stages, as shown in Figure 19. The upper and lower fracture branches reach (1) and breaks through the first interface (2) at the same time. But the stress distribution is no longer in symmetry from the reservoir top to bottom due to the influence of asymmetric mud interlayers. Afterwards, both upper and lower branches extend in the mud interlayer. But the lower branch quickly reaches the second 


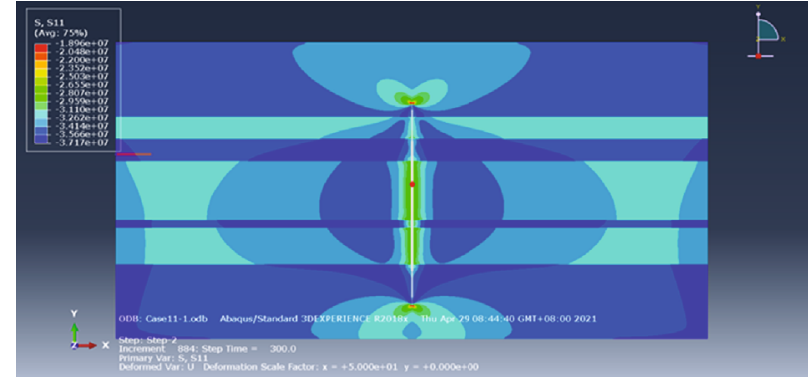

(a) Perforation above the model center

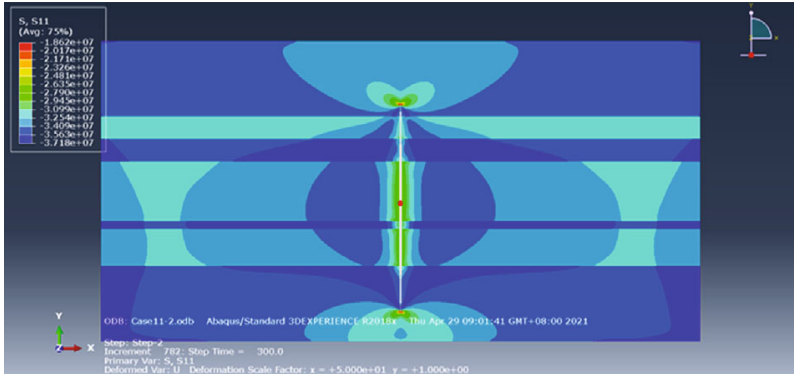

(b) Perforation below the model center

FIGURE 20: The influence of the perforation position on height growth.
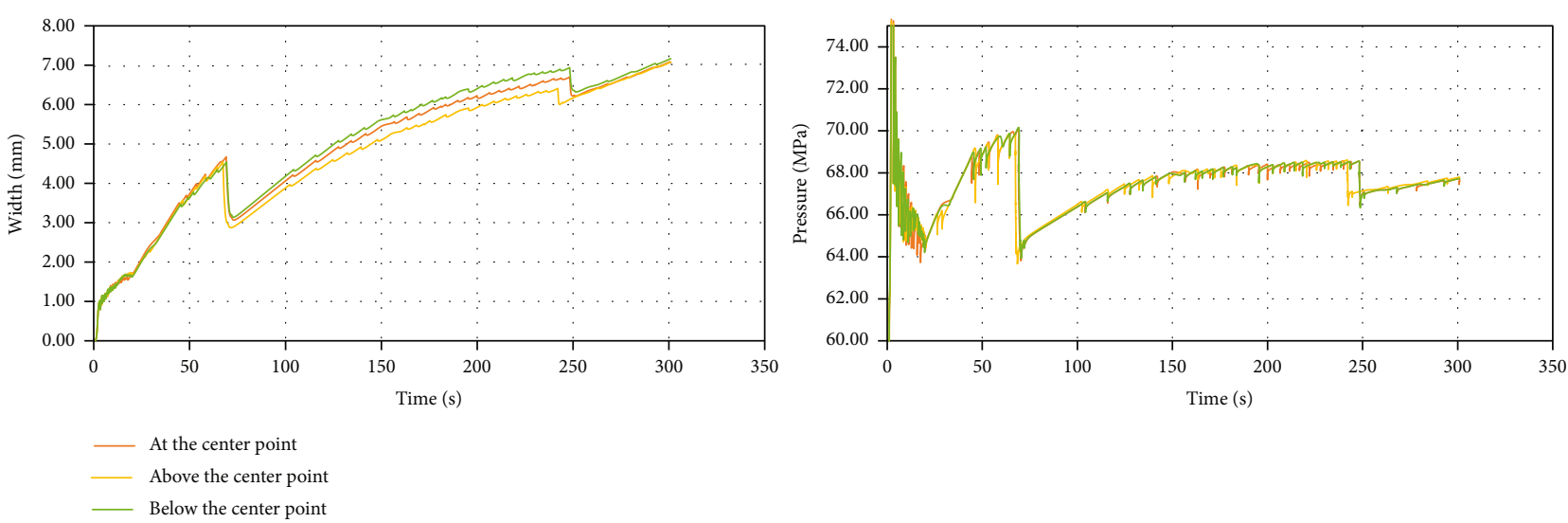

FIgURE 21: The influence of the perforation position on injection pressure and fracture width.

interface and the fracture width keeps growing (3). Right after the lower branch breaks through the lower thin mud interlayer, it goes quickly to the interface between sand and lower cap (4). Figure 18(c) shows at the moment when the lower branch reaches the cap interface, the upper branch is still inside the upper mud interlayer and does not break through. As both branches extend in mud, the pressure increases continuously. Finally, the upper branch breaks through the second interface and the width decreases immediately once more (5). As the number of mud asymmetric interlayers increases, the fluctuation of pressure and width will be more frequent. Every time the fracture breaks through the mud interlayer, it requires a higher pressure. Therefore, in reality, it may be difficult for the fracture to run through all interlayers. But at the early period, fluid with high viscosity and high rate is helpful for fracturing all sand reservoirs. Once the height extends into the multiple sand layers, it will become much easier for the fracture to propagate inside the sand.

In order to understand the influence of the perforation position on fracture height growth when the mud interlayers are asymmetric, two cases are studied. The distance between perforation to the center of the model in both Figures 20(a) and $20(\mathrm{~b})$ is $1 \mathrm{~m}$. In Figure 20(a), perforation is above the center of model. In Figure 20(b), perforation is below the center of the model. It can be observed that after both branches extend into the cap, these two cases have no difference from the perspective of stress filed. But during the propagation process, it can be observed in Figure 21 that when the perforation is below the center point, which means the position of perforation is close to the thinner mud interlayer, fracture width is much larger during fracture propagation. This is because the upper thick mud layer is difficult to break through and the fracture is asymmetric. That is to say, perforating close to the thinner mud layer can enlarge fracture width and make sand transport inside the fracture smoothly, which can reduce the risks of sand plugging.

\section{Conclusions}

This paper focuses on fracture height growth during hydraulic fracturing in the Dongsheng gas field. A hydromechanical sand-mud interbed model is established taking into full consideration reservoir characteristics. Besides, the parameters used in the simulations are from the experimental tests on downhole cores. Based on the numerical simulations, it can be concluded that fluid is difficult to flow inside the interfaces between sand and mud as it requires a much higher pressure to overcome overburden stress. As a result, the fracture extends vertically and breaks through mud interlayers without orientation. With a larger Young's modulus, fracture width in coarse sand is smaller than that in fine sand.

When there are mud interlayers within sand, the process of fracture height growth is complicated especially when the mud interlayers are asymmetric. Injection pressure and fracture width vibrate as fracture extends to and breaks through 
the mud interface. As the minimum horizontal stress difference between the mud interlayer and the sand layer increases, the net pressure required to break through the mud interlayer increases linearly. What is more, when the mud interlayers are asymmetric, the fracture branch near the thinner mud interlayer breaks through the interlayer and reaches cap interface first. Afterwards, the other side branch propagates to the cap interface as the pressure inside the fracture increases. What needs to be stressed is that what we studied in this paper is a $2 \mathrm{D}$ height growth problem. In a hydraulic fracturing, when fracture height extends to the mud interlayers, it may propagate continuously in sand rather than break through the mud interlayer. This is because the stress of mud is larger than that of sand under normal circumstance. Fracture propagates much easier horizontally. If we want the fracture height to break through multiple mud interlayers, a high net pressure inside the fracture is required. Therefore, according to the simulation results in this paper, high viscosity fluid and high injection rate are beneficial to build a high net pressure inside the fracture, especially at the early period. In addition, when the mud interlayers are asymmetric, perforation close to the thinner mud interlayer can enlarge fracture width and make sand transport inside the fracture smoothly, which can reduce the risk of sand plug.

\section{Data Availability}

The data presented in this paper are available upon request from the corresponding author.

\section{Conflicts of Interest}

The authors declare no conflict of interest.

\section{Acknowledgments}

This work was financially supported by the Sinopec Science and Technology project (P20065).

\section{References}

[1] Z. Lu, S. Ma, Y. He, and D. Niu, "Characteristics of sandy braided river intercalations in Ordos Basin: taking Sudong 27-36 tight well area for example," Fault-Block Oil \& Gas Field, vol. 25, no. 6, pp. 704-708, 2018.

[2] Z. Li, Y. Yang, J. Hou et al., "Reservoir architecture of turbidity channels in the 5th area of Bonan Oilfield," Journal of China University of Petroleum (Edition of Natural Science), vol. 39, no. 5, pp. 36-42, 2015.

[3] X. Xun, "Geological characteristics of Shihezi 1 member in Dongsheng gasfield and countermeasures against horizontalwell stimulation," Natural Gas Technology and Economy, vol. 13, no. 6, pp. 34-39, 2019.

[4] X. Xun, "Reservoir characteristics and classifica-tion evaluation of Shihezi 1 member, Jin 58 wellblock, Dongsheng Gasfield," Natural Gas Technology and Economy, vol. 12, no. 5, pp. 9-11, 2018.

[5] J. Cui, H. Li, J. Feng, C. N. Liu, and Y. J. Ju, "Barrier-beds and inter-beds characteristics and their effects on remaining oil distribution in braided river reservoirs," Special Oil \& Gas Reservoirs, vol. 20, no. 4, pp. 26-30, 2013.

[6] G. Irwin, "Relation of stresses near a crack to the crack extension force," in 9th Cong App Mech, Brussels, 1957.

[7] G. Irwin, "Analysis of stresses and strains near the end of a crack traversing a plate," Journal of Applied Mechanics, vol. 24, no. 3, pp. 361-364, 1957.

[8] G. I. Barenblatt, "The mathematical theory of equilibrium cracks in brittle fracture," Advances in Applied Mechanics, vol. 7, pp. 55-129, 1962.

[9] D. S. Dugdale, "Yielding of steel sheets containing slits," Journal of the Mechanics and Physics of Solids, vol. 8, no. 2, pp. 100-104, 1960.

[10] A. Hillerborg, M. Modeer, and P. Petersson, "Analysis of crack formation and crack growth in concrete by means of fracture mechanics and finite elements," Cement and Concrete Research, vol. 6, no. 6, pp. 773-781, 1976.

[11] M. Elices, G. Guinea, J. Gómez, and J. Planas, "The cohesive zone model: advantages, limitations and challenges," Engineering Fracture Mechanics, vol. 69, no. 2, pp. 137-163, 2002.

[12] Y. Li, J. Deng, W. Liu, and Y. Feng, "Modeling hydraulic fracture propagation using cohesive zone model equipped with frictional contact capability," Computers and Geotechnics, vol. 91, pp. 58-70, 2017.

[13] Y. Li, W. Liu, J. Deng, Y. Yang, and H. Zhu, “A 2D explicit numerical scheme-based pore pressure cohesive zone model for simulating hydraulic fracture propagation in naturally fractured formation," Energy Science \& Engineering, vol. 7, no. 5, pp. 1527-1543, 2019.

[14] Y. Li, J. Deng, W. Liu et al., "Numerical simulation of limitedentry multi-cluster fracturing in horizontal well," Journal of Petroleum Science and Engineering, vol. 152, pp. 443-455, 2017.

[15] Q. Gao and A. Ghassemi, “Three dimensional finite element simulations of hydraulic fracture height growth in layered formations using a coupled hydro-mechanical model," International Journal of Rock Mechanics and Mining Sciences, vol. 125, article 104137, 2020.

[16] K. Park, G. Paulino, and J. Roesler, "A unified potential-based cohesive model of mixed-mode fracture," Journal of the Mechanics and Physics of Solids, vol. 57, no. 6, pp. 891-908, 2009.

[17] K. Volokh, "Comparison between cohesive zone models," Communications in Numerical Methods in Engineering, vol. 20, no. 11, pp. 845-856, 2004.

[18] P. Camanho and C. Davila, Mixed-Mode Decohesion Finite Elements for the Simulation of Delamination in Composite Materials, NASA-Technical Paper, 2002.

[19] A. Taleghani, M. Gonzailez, and A. Shojaei, "Overview of numerical models for interactions between hydraulic fractures and natural fractures: challenges and limitations," Computers and Geotechnics, vol. 71, pp. 361-368, 2016.

[20] V. Tomar, J. Zhai, and M. Zhou, "Bounds for element size in a variable stiffness cohesive finite element model," International Journal for Numerical Methods in Engineering, vol. 61, no. 11, pp. 1894-1920, 2004.

[21] D. Simulia, Abaqus 6.13 User's Manual, Dassault Systems, Providence, RI, 2013.

[22] E. Detournay and H. Cheng, Fundamentals of Poroelasticity Comprehensive Rock Engineering: Principles. Practice \& Projects, Pergamon Press, Oxford, 1993. 
[23] Y. Wang, Y. Yi, C. Li, and J. Q. Han, “Anisotropic fracture and energy characteristics of a Tibet marble exposed to multi-level constant-amplitude (MLCA) cyclic loads: a labscale testing," Engineering Fracture Mechanics, vol. 244, article 107550, 2021.

[24] Y. Wang, B. Zhang, B. Li, and C. H. Li, "A strain-based fatigue damage model for naturally fractured marble subjected to freeze-thaw and uniaxial cyclic loads," International Journal of Damage Mechanics, vol. 30, no. 10, pp. 1594-1616, 2021. 